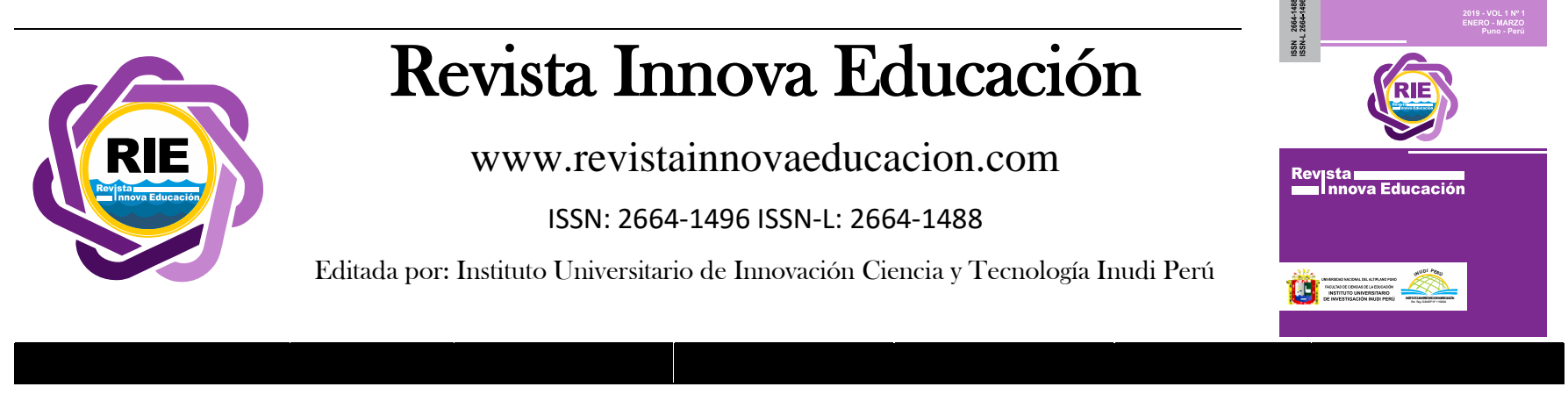

\title{
Diversificación curricular y la vigencia de la interculturalidad en los docentes de la Unidad de Gestión Educativa Local de Collao-Ilave
}

\section{Curricular diversification and the validity of interculturality in the teachers of the Unidad de Gestión Educativa Local of Collao-Ilave}

\author{
Katia Pérez iD) Tula Pérez; Luis Puño iD; Lesy León \\ DOI: https://doi.org/10.35622/j.rie.2019.01.010
}

Recibido el 15/01-2019/ Aceptado el 27/01/2019

\begin{tabular}{|c|c|}
\hline $\begin{array}{l}\text { ARTíCULO ORIGINAL } \\
\text { PALABRAS CLAVE } \\
\text { Aprendizaje, } \\
\text { currículo, } \\
\text { diversificación, } \\
\text { enseñanza, } \\
\text { interculturalidad. }\end{array}$ & $\begin{array}{l}\text { La investigación se realizó con el propósito de determinar la relación entre la } \\
\text { diversificación curricular y la vigencia de la interculturalidad en los docentes de la } \\
\text { UGEL }{ }^{1} \text { Collao - Ilave, respecto de las variables: diversificación curricular y la vigencia } \\
\text { de la interculturalidad. Metodológicamente, la investigación es de tipo descriptivo } \\
\text { correlacional; la población está constituida por } 50 \text { docentes, a quienes se les aplicó una } \\
\text { encuesta de } 20 \text { ítems para medir la variable diversificación curricular y se utilizó un } \\
\text { cuestionario para medir la variable vigencia de la interculturalidad y la diversificación } \\
\text { curricular en las instituciones de educación intercultural bilingüe de la zona rural. Para } \\
\text { establecer la correlación entre las variables diversificación curricular y la vigencia de } \\
\text { la interculturalidad, se utilizó el coeficiente R de Pearson. Los resultados de la } \\
\text { investigación son las siguientes: Existe una correlación positiva moderada entre la } \\
\text { diversificación curricular y la vigencia de la interculturalidad. Esta conclusión se } \\
\text { sustenta en la prueba estadística de Correlación de R de Pearson que es igual a 0.408; } \\
\text { lo que significa que a la existencia de una diversificación curricular se tendrá una } \\
\text { vigencia de la interculturalidad moderada; los docentes de la UGEL el Collao } \\
\text { consideran como vigente la interculturalidad en sus aulas. }\end{array}$ \\
\hline $\begin{array}{l}\text { Learning, curriculum, } \\
\text { diversification, } \\
\text { teaching, } \\
\text { interculturality. }\end{array}$ & $\begin{array}{l}\text { The research was carried out with the purpose of determining the relationship between } \\
\text { curricular diversification and the validity of interculturality in the teachers of the UGEL } \\
\text { Collao - Ilave, regarding the variables: curricular diversification and the validity of } \\
\text { interculturality. Methodologically, the research is descriptive correlational type; the } \\
\text { population is made up of } 50 \text { teachers, who were given a survey of } 20 \text { items to measure } \\
\text { the curricular diversification variable and a questionnaire was used to measure the } \\
\text { validity variable of interculturality. To establish the correlation between the variables } \\
\text { of curricular diversification and the validity of interculturality, the Pearson R } \\
\text { coefficient was used. The results of the research are the following: There is a moderate } \\
\text { positive correlation between curricular diversification and the validity of } \\
\text { interculturality. This conclusion is based on Pearson's statistical correlation test of R } \\
\text { that is equal to } 0.408 * * \text {; which means that the existence of a curricular diversification }\end{array}$ \\
\hline
\end{tabular}

${ }^{1}$ Unidad de Gestión Educativa Local 
will have a validity of moderate interculturality; the teachers of the UGEL Ilave consider interculturality valid.

\section{INTRODUCCIÓN}

El MINEDU (2009) afirma que los currículos básicos nacionales se diversifican en las instancias regionales y locales, en coherencia con las necesidades, demandas y características de los estudiantes y de la realidad social, cultural, lingüística, económico-productiva y geográfica en cada una de las regiones y localidades de nuestro país. Fernandez (2005) y Sáez, (2004) señalan que la diversificación curricular es entendida como un proceso de construcción a partir de las demandas, necesidades, intereses y características de los estudiantes y de la realidad socio - cultural y geográfica, local o regional. En este proceso el Diseño Curricular Nacional, es el referente que será adecuado, modificado y enriquecido de acuerdo a. la realidad. Para este proceso debemos tener en cuenta que no sólo es adecuar, sino de enriquecer, complementar, crear elementos curriculares dentro de un marco intercultural, tal como se menciona en el (MINEDU, 2016).

Un centro educativo intercultural debe contar con un proyecto integral de actuación que contemple todos los ámbitos educativos, éticos y sociales (Dicaudo, LLanos, \& Ospina, 2016). Los centros educativos deben transmitir que no podemos entender el discurso de la interculturalidad sin unos esquemas cognitivos y afectivos conectados entre sí; como también es insuficiente conocer las diversas culturas para una comprensión intercultural. Por eso, aumentar la sensibilidad intercultural conduce a aumentar la competencia intercultural, que incluye comunicación y actitudes (Williamson, 2004). En cambio en la escuela no es así, en ésta el niño indígena no puede entender el sentido del aprendizaje escolar a través de su experiencia inmediata, razón por la cual necesita de una motivación externa, según (MINEDU, 2013).

Los lineamientos generales que garantizan la diversidad y la cohesión de la sociedad peruana y sirven de base para diseñar currículos, propuestas o lineamientos regionales, según la decisión de dicho nivel de gobierno. El Currículo Nacional de Educación (CNE, 2006) propone que los programas de educación intercultural bilingüe están en demostrar la necesidad y viabilidad de una oferta diferenciada de calidad. Aseguran la pertinencia de los aprendizajes de acuerdo con la cultura y la lengua de cada población, así como con las diversas realidades sociales, económico» productivas y geográficas que tenemos en el país. Todo ello con el fin de garantizar el pleno desarrollo personal y social de los estudiantes. Para la realización de este proceso, se debe tener en cuenta el (MINEDU, 2009), elaborado por el Ministerio de Educación, el mismo que, para ser aplicado a cada realidad, debe ser/enriquecido y adecuado a las condiciones reales de cada Institución Educativa, en especial, a las necesidades de aprendizaje de los estudiantes y a los necesidades, intereses y aspiraciones ele la comunidad.

El (MINEDU, 2016) establece que la diversificación debe partir del diagnóstico de la problemática pedagógica de la Institución Educativa y, en función de ello, el Diseño Curricular Nacional es enriquecido y adecuado a las condiciones y modos de vida de los estudiantes, Todo ello con el fin de garantizar el pleno desarrollo personal y social de los estudiantes. Este proceso evidencia el respeto a la diversidad; por lo tanto, se ha de desarrollar considerando las culturas locales existentes en las regiones (expresiones culturales y naturales, potencialidades y problemas regionales, demandas de la sociedad y de los padres y madres de familia, intereses de aprendizaje y expectativas de los estudiantes) y las demandas de la sociedad peruana y mundial.

En América Latina y el Perú el concepto de interculturalidad, se introduce a inicios de la década de 1980 en los proyectos de educación bilingüe destinados a la población indígena como respuesta al fracaso de la educación asimilacionista y homogeneizante de los Estados nacionales, según (López \& Küper, 2000). Por otro lado, López (1988) nos dice que la escuela se convierte en punta de lanza de una ideología capitalista y de una visión occídentalizadora que ve en ella un instrumento indispensable para lograr ese ideal de homogeneidad lingüístico-cultural y económica que podría llevarnos hacia la tan ansiada unidad nacional.

El proceso de diversificación curricular se concretiza en la colaboración del Diseño Curricular Diversificado (DCD) del grado. Para este proceso debemos tener en cuenta que no sólo es adecuar, sino de 
enriquecer, complementar, crear elementos curriculares dentro de un marco intercultural, tal como se menciona en el (MINEDU, 2016).

Cruz (2015) publica el artículo: "La Interculturalidad en las Políticas de Educación Intercultural” en la revista Praxis situaciones sociales y personales \& Saber, en la investigación se concluye que, la educación intercultural ha sido pensada como una educación de tránsito, mientras los individuos de las culturas 'minoritarias' o sub alternizadas se adaptan a los valores y criterios de la cultura dominante". Boccara( 2012),en su investigación considera que: "La interculturalidad como campo social" en la revista Cuadernos Interculturales, en la investigación se concluye que, parece importante destacar es que los estudiosos que se dedican a buscar una definición correcta o precisa de lo que sería o debería ser intercultural son en realidad parte del juego social que pretenden explicar. Escarbajal (2014) en el estudio se concluye que, buscar enfoques afectivo-sociales para que todos los alumnos vivencien de comunicación intercultural; educar en valores que desarrollen la autonomía moral de los alumnos y el juicio crítico Tamayo (2016). en su investigación titulada: "Construcción del Currículo Tule - Ipkikuntiyala Antíoquia Colombia occidentales y viceversa., (Alvares ; Montaluisa, 2012) en su investigación titulada: "Comunidad, Escuela y Currículo" Santiago de Chile - UNESCO", en la que las labores y las discusiones del grupo se centraron en el intento de" con el único propósito de reafirmar la identidad cultural para posibilitar el amor a la madre tierra, a la conclusión principal que llegaron es que se debe aportar desde las concepciones, pedagogías y metodologías Tule, las concepciones, pedagogías y metodologías identificar una serie de criterios y condiciones generales y algunas propuestas específicas, que posibiliten la elaboración de currículos interculturales bilingües como medio para acercar la educación a las características y necesidades de los pueblos.

\section{MÉTODO Y MATERIALES}

En la presente investigación se utilizó la técnica de la encuesta y su instrumento el cuestionario; para la segunda variable, la técnica de análisis documental y su instrumento guía de análisis documental.

Se utilizó la técnica de encuesta y su instrumento de investigación fue el cuestionario. Los instrumentos de recolección de datos de la investigación fueron: Cuestionario para la diversificación curricular y Figura Clave de la Escala, calificaciones: Según ítems para cada área se suman los puntajes alcanzados y en total se convierte al puntaje normativo. La presente investigación es de tipo correlacional con una muestra intencional. La investigación tiene como objetivo determinar la relación entre dos variables asociativas y el cruce de dimensiones de ambas variables de estudio. Se fundamente en un enfoque cuantitativo de paradigma positivista y según criterio propósito es básico. El diseño que se utilizó para la presente investigación es descriptivo correlacional no experimental. Según (Oseda, 2008), el diseño no experimental o diseño ex post facto, son aquellos diseños donde las variables independientes no son manipuladas deliberadamente. Es correlacional porque precisa la relación entre dos variables. Para la recolección de datos se realizó a través de la aplicación de la encuesta para la variable diversificación curricular, que consta de 20 ítems. Para cada una de las alternativas se codificó, donde a cada nivel de escala se le da un valor respectivo, además para medir la diversificación curricular se realizó a través de la escala de Likert, que nos permitió ubicar los resultados obtenidos en la escala mencionada, que se detalla de la siguiente manera: Muy bajo, Bajo, Regular, Alto y Muy alto.

\section{RESULTADOS}

Diversificación Curricular: La investigación de esta variable se realizó con la escala de Likert. El proceso de recojo de datos se realizó con el instrumento señalado, se ejecutó en los meses de setiembre y octubre del presente año escolar. Se aplicó a una muestra constituida por 50 docentes de las instituciones educativas de nivel primarias de la UGEL Collao Ilave. Los datos tabulados considerando la escala de 
valoración de la variable respecto a la diversificación curricular se muestran en las siguientes tablas y figuras:

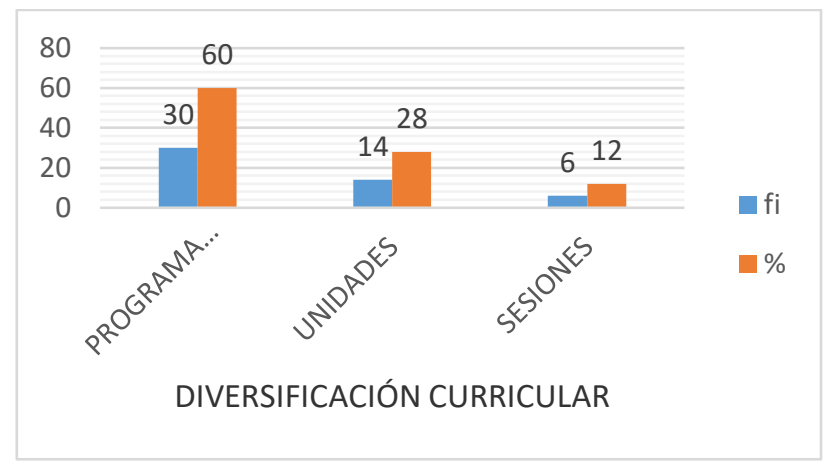

Figura 1: Nivel de frecuencia con la que diversifican de manera intercultural el programa curricular anual

En la figura 1 "Nivel de frecuencia con la que diversifican de manera intercultural el programa curricular anual" se evidencia la diversificación curricular en los docentes de la UGEL Collao - Ilave, datos que se ubican en el nivel alta y moderadamente respectivamente, y sólo en un porcentaje menor no se realiza adecuadamente la diversificación curricular.

Lo cual indica que los docentes de la UGEL Collao Ilave si realizan la inclusión de la interculturalidad en la: programación anual (60\%), unidades de aprendizaje (28\%) y sesiones de aprendizaje $(12 \%)$.

En la figura 1 también se observa que existe mayor porcentaje de diversificación en las programaciones curriculares anuales, a su vez se ve que sucede de manera contraria en las sesiones de aprendizaje.

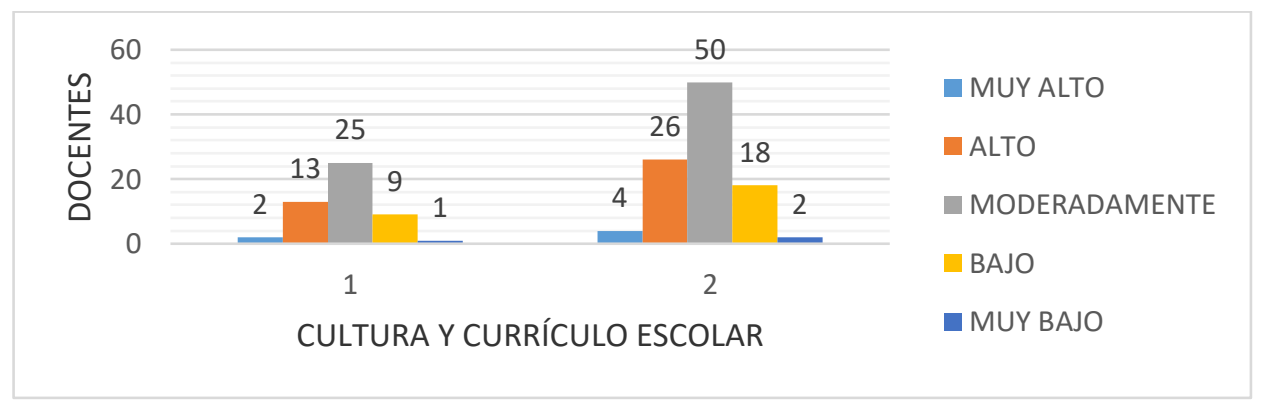

Figura 2. Nivel de relación entre cultura y currículo escolar

En la figura 2, se presentan los resultados sobre el nivel de relación entre cultura y currículo escolar. Los resultados muestran que en un $50 \%$ los docentes se ubican en el nivel moderadamente en el aspecto de la consideración de la cultura y currículo escolar. Sólo en un $2 \%$ los docentes tienen inconvenientes con respecto a tener inconvenientes en la consideración de la cultura y currículo escolar. En un nivel alto (26\%) se encuentran algunos docentes. En el nivel bajo (18\%) se ubican otros docentes y finalmente en el nivel muy alto $(4 \%)$ se encuentra una mínima cantidad de docentes. 


\section{Grado de correlación entre la diversificación curricular y la vigencia de la interculturalidad}

La diversificación curricular y la vigencia de la interculturalidad en los docentes de la UGEL Collao - Ilave, se midieron a través del sistema de correlación de Pearson en el formato estadístico de SPSS. A continuación, presentamos los resultados:

Tabla 1. Correlación entre La diversificación curricular y la vigencia de la interculturalidad en los docentes de la UGEL Collao - Ilave.

\begin{tabular}{ll|r|r}
\hline & \multicolumn{1}{c|}{$\begin{array}{c}\text { V. } \\
\text { VARIABLES ASOCIATIVAS }\end{array}$} & $\begin{array}{c}\text { V. VIGENCIA DE LA } \\
\text { DIVERIFICACIÓN } \\
\text { CURRICULAR }\end{array}$ & $\begin{array}{c}\text { INERCULTURALIDA } \\
\text { D }\end{array}$ \\
\hline V. DIVERSIFICACIÓN & Correlación de & 1 &, $408^{* *}$ \\
CURRICULAR & Pearson & &, 000 \\
& Sig. (bilateral) & 50 & 50 \\
& $\mathrm{~N}$ &, $408^{* * *}$ & 1 \\
V. VIGENCIA DE LA & Correlación de &, 000 & 50 \\
INTERCULTURALIDAD & Pearson & 50 & 5 \\
& Sig. (bilateral) & $\mathrm{N}$ & \\
& $\mathrm{N}$ & & 5 \\
\end{tabular}

Tabla 2: $\quad$ Valores del coeficiente de correlación

\begin{tabular}{c|l}
\hline VALOR & \multicolumn{1}{c}{ SIGNIFICADO } \\
\hline-1 & Correlación negativa grande y perfecta \\
\hline$-0,9$ a $-0,99$ & Correlación negativa muy alta \\
\hline$-0,7$ a $-0,89$ & Correlación negativa alta \\
\hline$-0,4$ a $-0,69$ & Correlación negativa moderada \\
\hline$-0,2$ a $-0,39$ & Correlación negativa baja \\
\hline$-0,01$ a $-0,19$ & Correlación negativa muy baja \\
\hline 0 & Correlación nula \\
\hline 0,01 a 0,19 & Correlación positiva muy baja \\
\hline 0,2 a 0,39 & Correlación positiva baja \\
\hline 0,4 a 0,69 & Correlación positiva moderada \\
\hline 0,7 a 0,89 & Correlación positiva alta \\
\hline 0,9 a 0,99 & Correlación positiva muy alta \\
\hline 1 & Correlación positiva grande y perfecta
\end{tabular}

Fuente: Karl Pearson

Representa que existe una correlación positiva moderada entre la diversificación curricular y la vigencia de la interculturalidad en los docentes de la UGEL Collao - Ilave.

\section{Planteamiento de hipótesis:}

Ho: $r=0$ No existe correlación entre la diversificación curricular y la vigencia de la interculturalidad en los docentes de la UGEL Collao - Ilave. 
Hi: /r/ > 0 Existe correlación entre la diversificación curricular y la vigencia de la interculturalidad en los docentes de la UGEL Collao - Ilave.

Nivel de significancia: $\alpha=5 \%$

\section{Regla de decisión:}

Entonces rechazamos la hipótesis nula y aceptamos la hipótesis alterna que indica que existe correlación entre entre la diversificación curricular y la vigencia de la interculturalidad en los docentes de la UGEL Collao - Ilave, dado que el coeficiente de correlación es de $\mathbf{r}=\mathbf{0 , 4 0 8}$ en un aprox. $40 \%$ con un margen de 0.05 .

Los resultados que hasta aquí se muestran, confirman las hipótesis planteadas en la investigación, es decir, existe una correlación de tipo directo entre la diversificación curricular y la vigencia de la interculturalidad en los docentes de la UGEL Collao - Ilave.

Respecto a los resultados a la correlación Los resultados de la investigación permiten sostener que la diversificación curricular y la vigencia de la interculturalidad existe un grado de correlación y de tipo directo. Este grado y tipo de correlación que se sustenta en el coeficiente de $\mathrm{r}=\mathbf{0 , 4 0 8}$, significa que cuanto mayor sea el nivel de diversificación curricular es mayor es el nivel de interculturalidad.

Además, se evidencia notablemente el paradigma positivista, lo que con los datos recogidos se demuestra objetivamente su veracidad. De tal manera que, el positivismo sustentado por Comte (1973) se demuestra con la presente investigación, porque se comprobó en hechos objetivos.

\section{DISCUSIÓN}

Los resultados de la investigación sobre la diversificación curricular y la vigencia de la interculturalidad en los docentes de la UGEL Collao Ilave, evidencian una correlación positiva moderada. Entonces se puede decir que la diversificación curricular se relaciona con la vigencia de la interculturalidad.

Con los resultados que se muestran en la tabla 6 y la figura 1 se prueba como cierta las hipótesis específicas planteadas, es decir, se relaciona moderadamente la diversificación curricular se relaciona con la vigencia de la interculturalidad en los docentes.

(Escarbajal Frutos, 2014; Vila \& Vega, 2012) indica que se debe buscar enfoques afectivo-sociales para que todos los alumnos vivencien situaciones sociales y personales de comunicación intercultural; educar en valores que desarrollen la autonomía moral de los alumnos y el juicio crítico; ello mediante actividades en las que se pongan encima de la mesa casos de dilemas morales, debates, asambleas, etc; utilizar los enfoques cooperativos, basados en la interdependencia, la responsabilidad y la ayuda mutua; hacer una exposición crítica de la realidad que sea comprensible por todos los alumnos, para que puedan así actuar responsablemente en la sociedad de manera transformadora; importante es también para un proyecto educativo intercultural la gestión democrática del centro, con la participación de las familias, una organización flexible de los espacios, el trabajo en equipo del profesorado, con elaboración propia de materiales didácticos y, cómo no, plantear el propio barrio y la escuela como recursos educativos.

La presente investigación evidencia gran importancia como la investigación de (Alvares, Catalina;Montaluisa, 2012) en la que advierte que las labores y las discusiones del grupo se centraron en el intento de identificar una serie de criterios y condiciones generales y algunas propuestas específicas, que posibiliten la elaboración de currículos interculturales bilingües como medio para acercar la educación a las características y necesidades de los pueblos. Y a la conclusión principal que llegó es que es importante denotar de esta investigación la necesidad de elaborar currículos jinterculturales bilingües acordes a las necesidades y características de los estudiantes en la vecina república de Chile, teniendo puntos en común ya que nuestras actuales políticas educativas reconocen la necesidad de una educación intercultural por tanto vemos los esfuerzos realizados pero que aún se tiene ciertas falencias de deben ser superadas con voluntad política y programas bien implementados. Encontrar el punto de equilibrio desde la perspectiva educativa, supone responder, desde el currículo escolar nacional, a las demandas de los pueblos originarios 
y afroperuanos y, al mismo tiempo, responder también a las demandas planteadas para la sociedad nacional y global.

Hasta ahora atender estas demandas educativas ha supuesto para el (MINEDU, 2017) un currículo nacional monocultural y ofrecer lineamientos para la diversificación curricular, esperando de esta manera otorgar pertinencia a los procesos pedagógicos en el aula. No obstante(Olmo, 2009) señala, que este esfuerzo, importante en un primer momento, resulta hoy insuficiente, pues se ha avanzado en la comprensión de que la Educación Intercultural debe ser para todos y que, tal como se expresa en la legislación nacional, debe plasmarse en todo el Sistema Educativo peruano. Primero, porque todos los estudiantes peruanos deben educarse para convivir armoniosamente y para construir una sociedad democrática, equitativa y justa; segundo, porque es legítimo que los pueblos originarios y afroperuanos que integran la nación peruana se sientan representados en el Currículo Nacional común. El justo equilibrio en esta doble condición es lo que permitirá la construcción de currículos locales pertinentes y solo de esta manera su formulación se hará desde y para la diversidad cultural (Abdallah, 2018; Dicaudo et al., 2016).

Por otro lado (Bravo, s. f.; Tamayo, 2016; Williamson, 2004), afirman que la educación intercultural ha sido pensada como una educación de tránsito, mientras los individuos de las culturas 'minoritarias' o subalternizadas se adaptan a los valores y criterios de la cultura dominante.(Vila \& Vega, 2012) Las políticas educativas formuladas como acciones afirmativas no tienen como fin mejorar los intercambios entre culturas, se concentran solamente en mitigar la desigualdad y la injusticia resultante de la dominación cultural, por eso sus acciones se focalizan en los grupos culturales minoritarios o subalternizados. Consiguientemente, las personas formadas en este marco tienen dos opciones: conservar los valores y entramados simbólicos de sus culturas, a riesgo de tener dificultades para dialogar con otros grupos culturales, $\mathrm{o}$ asimilarse a la cultura dominante.

\section{CONCLUSIONES}

Los resultados obtenidos de la presente investigación con respecto a nuestro objetivo general evidencian que existe correlación moderada entre la diversificación curricular y la vigencia de la interculturalidad en los docentes de las instituciones de la UGEL Collao del departamento de Puno, con un coeficiente de Pearson $r=0,408$. Se arriba a esta conclusión porque el $46 \%$ de docentes consideran que se realiza una diversificación curricular considerando la interculturalidad en la planificación y programación pedagógica.

Los docentes de las instituciones educativas del nivel primaria de la UGEL Collao diversifican de manera intercultural frecuentemente el programa curricular anual, los resultados demuestran la vigencia de la intercultural de manera moderada.

Los docentes de las instituciones educativas del nivel primaria de la UGEL Collao diversifican de manera intercultural frecuentemente las unidades de aprendizaje.

Los docentes de las instituciones educativas del nivel primaria de la UGEL Collao diversifican de manera intercultural con frecuencia las sesiones de aprendizaje.

\section{REFERENCIAS BIBLIOGRÁFICAS}

Abdallah, M. (2018). La educacion intercultural. Estudios sobre Educación, 3(0), 168.

Alvares, Catalina;Montaluisa, L. (2012). Educación, currículo y modos de vida: referentes para la construcción del conocimiento en el contexto Ecuatoriano, 2.

Boccara, G. (2012). La interculturalidad como campo social. Cuadernos Interculturales, 10(18), 11-30.

Bravo, K. del K. (s. f.). Atencion Pertinente a la Diversidad cultural en aulas del nivel inicial.

CNE. (2006). Proyecto Educativo Nacional al 2021.

Dicaudo, M., LLanos, D., \& Ospina, M. (2016). Interculturalidad y Educacion desde el Sur.

Katia Perez, Tula Pérez, Luis Puño, Lesy León 
Escarbajal, A. (2014). La educación intercultural en los centros educativos. Revista Electrónica Interuniversitaria de Formación del Profesorado, 17(2). https://doi.org/10.6018/reifop.17.2.197291

Escarbajal Frutos, A. (2014). La educación intercultural en los centros educativos. Revista Electrónica Interuniversitaria de Formación del Profesorado, 17(2). https://doi.org/10.6018/reifop.17.2.197291

Fernandez, F. (2005). Cuadernos interculturales. Cuadernos Interculturales, 3(4), 7.

López, L. E., \& Küper, W. (2000). La educación intercultural bilingüe en América Latina: balance y perspectivas.

MINEDU. (2009). Politica sectorial de educación intercuMINEDU. (2009). Politica sectorial de educación intercultural y educacion intercultural bilingue. Retrieved January 16, 2019, from http://repositorio.minedu.gob.pe/bitstream/handle/MINEDU/5960/Política sectorial de E.

MINEDU. (2013). Propuesta Pedagógica Hacia una Educación Intercultural Bilingüe de Calidad Hacia una Educación Intercultural Bilingüe de Calidad.

MINEDU. (2016). Curriculo Nacional de la Educacion Basica.

MINEDU. (2017). Programa curricular de Educacion Primaria.

Olmo, T. A. M. del. (2009). Educacion Intercultural,Perspectivas y Propuestas.

Oseda. (2008). Diseño de la investigacion. Lima, Peru.

Sáez, R. (2016). La educacion intercultural. Revista de Educación, (339), 859-881. Recuperado de http://www.revistaeducacion.mec.es/re339/re339a37.pdf

Tamayo, C. (2016). Curriculo escolar, conocimiento matematico y practicas sociales: posibilidades otras en una comunidad indigena gunadule. Tamayo, C. I. (n.d.). School curriculum, [mathematical] knowledge and social practices: other possibilities in an indigenous Gunadule community, 4, 903919. https://doi.org/10.1590/S1517-9702201612145827

Vila, E., \& Vega, F. (2012). La educacion para la autonomia moral en la escuela intercultural. Teoria de la autonomia moral en la escuela intercultural, 13(1138-9737).

Williamson, G. (2004). Cuadernos interculturales. Cuadernos Interculturales, 2(3). 